\title{
Phronesis
}

\section{Des pratiques pédagogiques de l'enseignement du lire/écrire, déclarées par des enseignantes du primaire Des pratiques pédagogiques de l'enseignement du lire/écrire, déclarées par des enseignantes du primaire}

\author{
Rakia Laroui, Magalie Morel et Stéphanie Leblanc
}

Volume 3, numéro 1-2, janvier-avril 2014

Le stage en formation, tendances et résistances

URI : https://id.erudit.org/iderudit/1024594ar

DOI : https://doi.org/10.7202/1024594ar

Aller au sommaire du numéro

Éditeur(s)

Université de Sherbrooke

ISSN

1925-4873 (numérique)

Découvrir la revue

Citer cet article

Laroui, R., Morel, M. \& Leblanc, S. (2014). Des pratiques pédagogiques de

l'enseignement du lire/écrire, déclarées par des enseignantes du primaire.

Phronesis, 3(1-2), 111-120. https://doi.org/10.7202/1024594ar
Résumé de l'article

Plusieurs élèves de niveau primaire présentent des difficultés de lecture et d'écriture. Or, la recherche montre que les pratiques enseignantes efficaces ont une incidence favorable sur le succès des élèves dans leur apprentissage du lire-écrire. Suivant une recension des écrits sur les pratiques gagnantes au regard des théories de l'apprentissage socioconstructivistes, cet article présente les résultats d'une recherche portant sur les pratiques déclarées de l'enseignement du lire-écrire de quatre enseignantes de sixième année du primaire. Les résultats montrent que bien que les enseignantes interrogées utilisent encore des pratiques d'enseignement traditionnelles telles que la dictée, elles utilisent généralement des pratiques novatrices qui intègrent les enseignements de la lecture, de l'écriture et de la communication orale. Elles mettent également de l'avant des projets interdisciplinaires et transversaux. Les activités proposées sont de plus signifiantes, car connectées sur la réalité et les intérêts des élèves. En somme, les enseignantes interrogées révèlent que leur enseignement du français est décloisonné et constitue un tout, tel que le recommandent les études sur les pratiques efficaces. 


\title{
Des pratiques pédagogiques de l'enseignement du lire/écrire, déclarées par des enseignantes du primaire
}

\author{
Rakia LAROUI, Magalie MOREL et Stéphanie LEBLANC
}

\begin{abstract}
Université de Rimouski
300 Allée des Ursulines

Rimouski (Québec)

Canada G5L 3A1

rkialaroui@uqar.ca
\end{abstract}

Mots -clés : enseignement du lire-écrire au primaire, pratiques pédagogiques déclarées, théories de l’apprentissage socioconstructivistes, approche intégrée de l'enseignement du lire-écrire.

Résumé : Plusieurs élèves de niveau primaire présentent des difficultés de lecture et décriture. Or, la recherche montre que les pratiques enseignantes efficaces ont une incidence favorable sur le succès des élèves dans leur apprentissage du lire-écrire. Suivant une recension des écrits sur les pratiques gagnantes au regard des théories de l'apprentissage socioconstructivistes, cet article présente les résultats d'une recherche portant sur les pratiques déclarées de l’enseignement du lire-écrire de quatre enseignantes de sixième année du primaire. Les résultats montrent que bien que les enseignantes interrogées utilisent encore des pratiques d’enseignement traditionnelles telles que la dictée, elles utilisent généralement des pratiques novatrices qui intègrent les enseignements de la lecture, de lécriture et de la communication orale. Elles mettent également de l'avant des projets interdisciplinaires et transversaux. Les activités proposées sont de plus signifiantes, car connectées sur la réalité et les intérêts des élèves. En somme, les enseignantes interrogées révèlent que leur enseignement du français est décloisonné et constitue un tout, tel que le recommandent les études sur les pratiques efficaces.

\section{Title: Des pratiques pédagogiques de l'enseignement du lire/écrire, déclarées par des enseignantes du primaire}

Key-words: reading and writing teaching in elementary school, reported formative practices, social constructivist learning theories, reading and writing teaching's integrated approach.

Abstract: Many elementary school students have difficulties in reading and writing. However, researches showed that effective teaching practices have positive impacts in the success of students at school precisely in their reading and writing learning process. This paper presents results of a study on reported reading and writing teaching practices conducted on four sixth grade teachers by following a literature review on social constructivist theories winning practices. The results showed that they use innovative practices that incorporate reading, writing and oral communication teachings in class; even if they still have traditional practices such as dictation. They also focused on interdisciplinary and crossfunctional projects. Those activities are more significant because of their closeness with the children's reality and interests. In sum, the interviews revealed the teachers' methods as a whole and an open-plan, which many effective practices studies recommend. 
Dans un contexte où l'apprentissage du lire-écrire au primaire est une problématique centrale au Québec et ailleurs, il importe de s'interroger sur les pratiques enseignantes ainsi que sur les approches privilégiées quant à l'enseignement et l'apprentissage du lire-écrire. Ce texte présente quelques éléments de la problématique de l’apprentissage et de l'enseignement du français au primaire. Il expose également, en recension des écrits, les résultats des recherches sur l'impact des pratiques enseignantes sur le processus d’apprentissage du lire-écrire auprès des élèves du primaire. Le cadre théorique qui supporte ces recherches s'inspire particulièrement de théories d'apprentissage socioconstructivistes. Ensuite, l’article expose les résultats d’une étude exploratoire menée à l’aide d’entrevues semi-dirigées auprès de quatre enseignantes de la sixième année du primaire dans la région du Bas-Saint-Laurent au Québec. Létude qualitative vise à identifier les pratiques pédagogiques d’enseignement du lire-écrire utilisées par les quatre enseignantes participantes. Lanalyse qualitative des données nous oriente vers l’efficacité et la nécessité de la pratique des approches intégrées en enseignement, pour aider lélève dans son apprentissage. Enfin, quelques pistes de réflexion sont évoquées en conclusion.

\section{Problématique}

Plusieurs élèves du primaire éprouvent des difficultés d’apprentissage en lecture (Giasson, 2011) et en écriture (Debeurme, 2006) ce qui entraine des conséquences tout au long du parcours scolaire (Morin et Montésinos-Gelet, 2007; Nadon, 2002) pouvant mener à léchec et au décrochage scolaire. Compte tenu de ces difficultés d’apprentissage, le projet d’apprendre à lire et à écrire risque de s’affaiblir chez lélève au point de le mettre en situation d'échec (Goupil, 2007). Puisque la lecture et lécriture sont impliquées dans tous les domaines d’apprentissage, cela représente un risque réel déchec et d’abandon scolaire (Van Grunderbeeck, Théôet, Chouinard et Cartier, 2003).

Dès 1999, le Conseil supérieur de léducation (CSE) soulignait qu’un pourcentage élevé délèves ne possède pas les compétences pour accéder au secondaire et les données du Ministère de léducation, du loisir et du sport (MELS, 2008) soulèvent des constats problématiques quant à la réussite des élèves : $25 \%$ des élèves de la $6^{\mathrm{e}}$ année du primaire se trouvent en situation à risque. En effet, les résultats aux épreuves terminales en écriture en $6^{e}$ année montrent une augmentation du nombre délèves en situation déchec entre 2000 et 2005, passant ainsi de $10 \%$ à $20 \%$. Notons également que ces mêmes données (MELS, 2005) soulignent que le rendement spécifique en orthographe a connu une baisse importante passant de $8 \%$ à $26 \%$ entre 2000 et 2005. Le taux d'échec à lépreuve obligatoire décriture demeurait élevé en 2009 et 2010, soit respectivement de $24,7 \%$ et de $20,6 \%$

Ces difficultés éprouvées en français par les élèves ainsi que le retard scolaire qu'ils accusent à l’arrivée du secondaire posent des questions quant à la réussite et la persévérance des élèves.

Depuis quelques années, la recherche montre la nécessité de changer les pratiques enseignantes pour aider les élèves dans leur apprentissage du lire-écrire. Des mesures pédagogiques dont la lecture et l'écriture régulières des élèves sont proposées. Néanmoins, elles ne constituent pas une pratique habituelle des enseignants selon le rapport du Ministère de léducation, du loisir et du sport (2008). Des recherches relatives à lobservation de pratiques enseignantes (Strickland, Ganske et Monroe, 2009 ; Prenoveau, 2007) décrivent la complexité du rapport qu'entretient l'enfant avec lécrit tout au long du primaire et affirment que des pratiques pédagogiques exemplaires pourraient faciliter le développement des compétences à lire et à écrire chez les élèves. Selon Prenoveau (2007), afin de cultiver le goût de lire et d'écrire, il faut enseigner la lecture et lécriture par une approche intégrée et équilibrée entre plusieurs activités. Les pratiques pédagogiques ainsi devraient exploiter des activités diversifiées : lecture collaborative, interactive et écriture partagée, guidée, modélisée, autonome. Pour Prenoveau (2007), les élèves, dès le début du primaire, devraient lire et écrire quotidiennement. De plus, dès la première année du primaire ainsi qu’à la sixième année du primaire, classes charnières dans l’apprentissage de l'écrit, les élèves devraient réaliser des projets d'écriture transversaux. Ces travaux ouvrent des perspectives privilégiant des séquences d’enseignement articulant la lecture et l'écriture dans le cadre d’approches 
intégrées de l’enseignement du lire-écrire. D’autres recherches (Adad et Richard-Principalli, 2007 ; Giasson, 2011) indiquent que les pratiques gagnantes en enseignement reposent principalement sur la compétence des enseignantes. Ces pratiques gagnantes, ou exemplaires, doivent privilégier des activités qui intègrent la lecture et lécriture ainsi que la communication orale selon une démarche bien définie aux élèves leur rendant explicites les stratégies de lecture, décriture et de communication orale. Reuter (2000, 2011) et Routman (2009) soutiennent qu'il existe une corrélation entre les performances des élèves en écriture et en lecture et les pratiques enseignantes gagnantes. Ils considèrent que ces pratiques jouent un rôle important et peuvent aider les élèves, même en difficulté, vers un apprentissage réussi du lire-écrire. Le MELS (2008) rappelle que l’acte d'écrire, compétence à développer, est une tâche complexe :

[...] c'est beaucoup plus quécrire un texte sans faute. Écrire constitue un acte complexe qui met en jeu à la fois des processus cognitifs et des représentations sociales. Lélève doit planifier sa démarche, mettre son texte en forme et le réviser. Il a donc beaucoup d’aspects à gérer en plus de la grammaire. (p. 4)

En somme, les précédentes études montrent que l’apprentissage de la lecture et de lécriture à travers des activités motivantes et signifiantes dans un environnement scolaire constructif offre un support aux élèves. Ajoutons que dans cet environnement, les élèves travaillent en équipe pour s'entraider. Ils lisent pour écrire et ils écrivent en réponse aux textes lus. Les activités sont signifiantes puisqu’elles répondent à des objectifs clarifiés dans des tâches qui intègrent la lecture et l'écriture. Ainsi, l’enseignement de ces compétences essentielles à l'école et dans la vie de tous les jours est modélisé et explicité afin d’améliorer les connaissances des élèves. Tout en utilisant une variété de procédures en lecture et en écriture, l’enseignement intègre aussi les rétroactions des élèves. La prise en compte de ces éléments dans lélaboration d'une démarche d’enseignement de la lecture et de l'écriture au primaire serait à privilégier selon les recherches citées auparavant.

Les différentes recherches citées montrent également que les pratiques enseignantes exercent une influence majeure dans le développement des compétences et des savoirs chez les élèves afin de les mener à la réussite dans leurs apprentissages. Ceci est particulièrement important dans la réussite au plan du lire-écrire. Il apparaît que peu d’études ont été réalisées sur les pratiques enseignantes déclarées en $6^{e}$ année du primaire alors que cette période se veut particulièrement importante pour les élèves. En effet, la $6^{\mathrm{e}}$ année annonce la fin du niveau primaire vers le passage au secondaire. Les lacunes au niveau des compétences et des savoirs des élèves à ce moment charnière de leur parcours scolaire peuvent avoir des conséquences importantes quant au risque d’échec et d’abandon scolaire. Ces quelques éléments de problématique nous mènent à la nécessité d’examiner quelles sont les pratiques enseignantes utilisées en $6^{e}$ année du primaire en français.

\section{Cadre de référence}

\subsection{Théorie d’apprentissage socioconstructiviste}

La recherche au sein de la communauté scientifique tend à démontrer que des pratiques enseignantes considérées efficaces dans le cadre de l'enseignement de la lecture et de lécriture aident les élèves dans leur développement langagier.

Les auteurs des recherches citées et exposées ci-dessus s'inspirent en général de la théorie d’apprentissage socioconstructiviste, élaborée par Vygotski (1985). Les aspects théoriques et pratiques de la portée de son œuvre montrent l'intérêt que porte cet éducateur et psychologue aux rapports complexes entre le développement de l'enfant et l'apprentissage langagier. Vygotski a montré l'impact positif des interactions entre pairs comme mode d'apprentissage. Ces interactions facilitent chez l'enfant la construction des connaissances. Ainsi, lors de tâches exigeantes et complexes, les dialogues collaboratifs et coopératifs entre les pairs et avec l'adulte enseignant stimulent l'apprentissage de la langue et la pensée chez l'enfant. Selon Lavoie, Lévesque et Laroui (2007), la tâche de médiation de l’enseignant est primordiale. Cette tâche de médiation de l’enseignant qui doit interagir avec lélève pour le supporter et l'accompagner dans son apprentissage constitue un concept clé dans la théorie d’apprentissage socioconstructiviste. Bertrand (1993) analyse le modèle socioconstructiviste et précise que l'apprentissage est un phénomène social et culturel et que l’enfant apprend comme on lui a enseigné. Le comportement verbal de l'apprenant est déterminé par le contexte de l’apprentissage. Pour Vygotski, l'apprentissage de la langue écrite suppose un regard sur la langue parlée qui est de lordre du métacognitif. Pour apprendre à lire et à écrire, des correspondances possibles entre les unités de la langue orale et celles de la langue écrite sont utiles. De plus, le rôle des interactions sociales dans l'apprentissage est primordial. Actuellement, il est presque impossible dans le domaine de léducation d'isoler, en apprentissage, l'acte propre du sujet qui apprend de l'activité de médiation de l'enseignant. 


\subsection{Recherches actuelles en didactique du lire-écrire}

La recherche en didactique du français aujourd'hui (Giasson, 2011; Laroui, 2008, 2010 ; Lefrançois, 2000 ; Reuter, 2011) prend en considération les concepts clés du cognitivisme (Tardif, 1997) et des théories d'apprentissage socioconstructivistes pour analyser, dans l'apprentissage, l'activité conjointe des élèves entre eux et de l’enseignant avec les élèves.

Plusieurs pratiques enseignantes gagnantes ont été résumées par Hall (2003). L'accent est mis sur les approches intégrées de l’enseignement de la lecture et de l'écriture ainsi que sur un apprentissage formel et explicite de la langue. De plus, ces pratiques exemplaires cherchent à développer la réflexivité des élèves par rapport à la langue et aux stratégies qu'ils déploient pour comprendre et produire des textes. Elles prennent explicitement appui sur les milieux culturels des enfants. Ces pratiques mettent l'accent sur la différenciation en fonction des besoins des élèves. Elles placent l'élève dans un contexte interactif d’apprentissage (Blain et Lafontaine, 2010) et privilégient l'enseignement explicite (Bissonnette, Richard et Gauthier, 2005) pour mener plus loin dans leurs apprentissages les élèves. Les enseignants qui permettent à leurs élèves de développer leurs compétences en lecture et en écriture de manière optimale enseignent explicitement et par application concrète les stratégies de reconnaissance de mots, le vocabulaire, lorthographe, les stratégies de compréhension de textes ainsi que les processus décriture. De plus, selon Hall (2003), l'intégration lecture/écriture offre une plus grande variété de tâches aux élèves. Ils font de la lecture indépendante et individuelle, mais aussi en tandem, en groupe classe ainsi que de lécriture collaborative pour une variété de motifs. Ces tâches exigeantes cognitivement sont formatrices pour les élèves. De plus, les enseignants modélisent des comportements de lecteurs et de scripteurs initiés et cherchent à développer la réflexivité des élèves par rapport à la langue et aux stratégies qu'ils déploient pour comprendre et produire des discours.

Ces pratiques exemplaires tiennent compte des besoins différenciés des enfants. Elles font une exploitation judicieuse de la littérature de jeunesse dans les classes. Celle-ci se veut une source d'inspiration pour lélaboration de diverses activités d'apprentissage qui intègre la lecture, l'écriture et la communication orale (Pasa, Raganon et Filjalkow, 2006). Le Programme de formation de lécole québécoise (PFEQ) (MEQ, 2001), s'inspirant des théories d’apprentissage cognitiviste et socioconstructiviste, privilégie clairement une approche intégrée du lire-écrire. Ces éléments de problématique ainsi que les résultats de recherche cités plus haut orientent notre étude vers lobjectif suivant : Identifier les pratiques pédagogiques déclarées par des enseignantes de $\sigma^{e}$ année du primaire en enseignement/apprentissage du lire-écrire.

\section{Méthodologie de recherche}

La méthode de recherche privilégiée est l'approche qualitative de collecte et d'analyse des données recueillies auprès de quatre enseignantes du primaire. Cette approche de recherche qualitative interprétative vise à dégager des pistes de réflexion à partir des expériences des participantes. Le petit nombre d'enseignantes permet d'étudier en profondeur leurs pratiques. Des entrevues ont été réalisées à l'hiver et au printemps 2012 au sein de la Commission scolaire des Phares, située dans le Bas-Saint-Laurent (Québec). L'entrevue semi-dirigée, qui s'est effectuée dans la classe même où ouvre l’enseignante interrogée, se présente en deux parties principales pour susciter la discussion. La première partie porte sur les pratiques et les méthodes employées par les enseignantes alors que la seconde partie les interroge sur ce qui caractérise leurs élèves. Ces enseignantes possèdent entre 5 et 20 ans d'expérience, majoritairement acquise à l'ordre primaire. À l'aide d'un canevas d’entrevue semi-dirigée (annexe 1), composé de questions ouvertes (Savoie-Zajc, 2002), les enseignantes discutent de leurs pratiques en classe afin d’amener les élèves à faire l'apprentissage du français, surtout dans la pratique de l'écriture. Enregistrées sur support numérique, les entrevues sont transformées en verbatim pour être analysées qualitativement à l'aide du logiciel d’analyse qualitative NVivo 9 selon la procédure d'analyse de contenu (Landry, 2002). Une grille d’analyse thématique, construite d'une part à l'aide de la littérature produite dans le domaine de la didactique du français et d'autre part par l'émergence des thèmes tout au long de l'analyse même des entrevues, soutient ce processus. Cette procédure permet ainsi de relever et de quantifier l’apparition de thèmes dans le corpus de données. La grille d’analyse, une fois son élaboration terminée, est appliquée de nouveau à l'ensemble du corpus afin de s’assurer que tous ses éléments ont été analysés adéquatement (Mucchielli, 2009).

\section{Présentation et analyse des résultats}

Les résultats présentés portent sur quelques caractéristiques des enseignantes puis sur les méthodes et les pratiques d'enseignement mises en place en classe. 
Les données analysées montrent que lévolution des pratiques d'enseignement joue un rôle primordial dans l'intérêt porté à la profession. En effet, « Je me suis dit : "ça n’a pas de bon sens, on ne donne pas le goût aux jeunes d’écrire et lire”. [...] j’ai eu deux choix : ou j’abandonne [...] ou je fais de la recherche et je modifie ma façon d'enseigner. » (Enseignante 2)

Il s'avère aussi important pour les enseignantes de se présenter comme un modèle de scripteur devant les élèves pour les encourager à écrire. "J'ai écrit un manuscrit, donc je sors toujours mon manuscrit puis je leur dis bon : "Regardez, j’ai appris à écrire des belles phrases". » (Enseignante 1) De plus, une relation de confiance doit sétablir avec les élèves : « [...] il faut qu’ils me fassent confiance [...] pour arriver à bien écrire. » (Enseignante 3)

Un des éléments que fait ressortir l'enseignante 1 est de développer le plaisir de l'écriture chez les élèves. C’est pourquoi elle choisit, dans les pratiques d'écriture, des tâches et des consignes permettant aux élèves de développer leur imagination avec des sujets qui les intéressent. Une enseignante ajoute qu’en plus d’apprendre le français avec rigueur, le plaisir de jouer avec les mots est également nécessaire.

\subsection{Méthodes et pratiques employées}

Les enseignantes rencontrées emploient une variété de méthodes et de pratiques pour amener les élèves à développer leurs connaissances et leurs compétences en écriture.

D’abord, un des éléments importants est la signifiance des apprentissages. Il faut amener les élèves à comprendre pourquoi le français est utile et pertinent. Pour l'enseignante 1, la signifiance réside dans l'utilisation de l'actualité ou dans l'exploitation des champs d'intérêt les élèves. « [...] je propose toujours des sujets libres. C’est incroyable les sujets que j’ai. Variés, intéressants, j’en ai toujours sur les animaux [...] un thème scientifique $[\ldots]$ une invention $[\ldots] »$ (Enseignante 1 ).

L'enseignante 3 indique « [...] que c'est donner de l'importance aussi à ce qu'ils ont à produire. Ce n'est pas juste pour la note [...]. » La signifiance se rattache aussi aux besoins manifestés par les élèves. Ainsi, elle les amène à écrire « [...] des lettres, des résumés, des textes où ils doivent donner leur opinion. [...] ». L’enseignante 4 propose différentes activités, telles que la production d'un journal diffusé dans toute lécole, de rédiger des textes pour des affiches qui sont accrochées sur les murs de lécole, etc. Bref, la production de textes lus par différents acteurs.

Plusieurs formes d'écriture sont abordées dans les classes : poésie, bande dessinée, texte déclaratif, texte d’opinion, conte et légende, affiche publicitaire, etc. Lécriture peut être associée à la lecture par la production de résumés de lecture : « [...] je leur fais faire un résumé pour qu'ils continuent à pratiquer leur écriture [...]. » (Enseignante 3) L'enseignante 4 intègre aussi une activité de type «improvisation écrite » pour placer les élèves en situation décriture.

Lapprentissage de la langue écrite suppose l'acquisition des règles de grammaire, d’orthographe et de syntaxe. Différentes mesures sont mises en place. L’enseignante 3 s'appuie sur l'étude des classes de mots et sur lélaboration d'un lexique afin d'aider les élèves à retenir des normes grammaticales.

Pour l'enseignante 2, il existe de multiples occasions d’apprendre de nouvelles notions sans qu'elles soient prescrites dans le programme. Par exemple, le recours à la dictée Paul-Gérin-Lajoie (PGL) l’amène à enseigner l’accord des participes passés.

L'exploitation de la littérature jeunesse constitue un autre outil pour amener les élèves à développer leur goût pour la lecture et lécriture. La présence de bibliothèques proposant des collections adaptées à la clientèle de $6^{\mathrm{e}}$ année (Les chats de gouttière ou Les Maboules) et des activités comme le cercle littéraire contribuent au développement de différentes compétences reliées à la lecture, à lécriture et à l'expression orale. Depuis plusieurs années, les technologies de l'information et de la communication TIC, comme lordinateur ou le tableau interactif (TBI), sont présentes dans les écoles du Québec. L’enseignante 1 a déjà organisé une correspondance scolaire par Internet, mais c'est une pratique qu’elle a délaissée puisqu’elle souhaite que ses élèves écrivent à la main pour développer le plaisir de la calligraphie.

Le dictionnaire demeure un outil fondamental et son exploitation en classe varie selon l'enseignante et leur disponibilité. Dans certains cas, chaque élève dispose d'un dictionnaire alors quailleurs, il s’agit d'un usage partagé. L'enseignante 1 indique l'utiliser quotidiennement. Lenseignante 3 indique que les élèves doivent identifier dans leurs pratiques d'écriture un certain nombre de mots qu'ils sont allés chercher dans le dictionnaire. En plus des dictionnaires classiques (Larousse, Robert), les élèves utilisent le dictionnaire Euréka ${ }^{1}$. De son côté, l'enseignante 4 l'exploite comme outil de référence.

En plus du matériel pédagogique et didactique disponible dans les écoles, les enseignantes développent du matériel qui leur est propre pour adapter leurs pratiques pédagogiques aux élèves. L’enseignante 4 a mis en place un journal de bord avec ses élèves afin de les amener à écrire tout au long de l’année scolaire à partir de questions variées et de thématiques diversifiées.

1 Le dictionnaire Euréka est un dictionnaire dans lequel les mots sont classifiés selon une transparence orthographique en début de mot. Par exemple, le mot pharmacie serait classifié dans les $\mathrm{f}$. 
Des pratiques plus classiques telles que la dictée occupent une place encore importante. Il existe plusieurs types de dictées : dictée formelle, dictée de mots, dictée Zéro faute, etc. Pour l'enseignante 1, la dictée permet un retour sur les notions de grammaire. Elle propose aussi aux élèves la dictée Zéro faute qu’elle décrit comme suit :

[...] cést une dictée qui te donne la possibilité de poser des questions à ton professeur et de douter de mots. Puis je peux prendre un extrait de n’importe quoi [...] Puis après ça, ils ont le droit de me poser des questions. [...] Moi jai le droit de répondre [...] Puis, jajoute une notion de grammaire. [...] je leur dis souvent quand je donne des dictées, mon but ce n'est pas d'ensanglanter les copies [...], mais de donner le doute, que l'enfant écrive [...] qu'il doute de l'orthographe [...] qu'il se pose la question pourquoi cést comme ça [...] qu'ils se questionnent au niveau de la grammaire. [...] ils ont l'impression que je leur donne les réponses, mais je ne leur donne pas les réponses, je les amène à se questionner. [...] j’y crois beaucoup aux dictées. (Enseignante 1)

L’enseignante 4 effectue une dictée chaque vendredi afin de vérifier l'acquisition des notions étudiées durant la semaine (vocabulaire, homophones, etc.). La méthode de dictée Zéro faute, également employée dans sa classe, est appréciée par les élèves : « Ils aiment ça beaucoup [...] ils ont comme l'impression que c'est eux le professeur, le fait qu'ils posent une question. » (Enseignante 4)

Les équipes-écoles développent aussi des stratégies pour soutenir les enseignantes. En effet, l’enseignante 3 explique que la continuité est importante pour les élèves. Dans un milieu divisé en deux pavillons, léquipe-école était confrontée à des problèmes. Des solutions sont mises en place pour : « [...] se donner le même langage au niveau des classes de mots [...] de la façon dont on travaille les adjectifs pour que [...] lélève n'ait pas un autre apprentissage à faire l’année d’après $[\ldots] »$. (Enseignante 3) Ce travail déquipe assure une cohérence dans les apprentissages des élèves durant le parcours scolaire au primaire.

\subsection{Les stratégies d'enseignement et d'apprentissage}

Les enseignantes emploient une grande variété de stratégies d’enseignement et d’apprentissage pour mener les élèves à atteindre les objectifs prévus au programme de formation.

La pédagogie différenciée occupe une place importante dans les préoccupations des enseignantes confrontées à une clientèle scolaire variée. L’enseignante 1 pratique la pédagogie différenciée lors de lévaluation des élèves. Elle donne l’exemple d’un test portant sur les verbes. « Le groupe 02 réussit beaucoup mieux leur test de verbes, le groupe 01, je réussis par leur demander à l'oral. Le conditionnel présent du verbe aimer, ils sont capables à loral facilement, ils réussissent tous, mais quand je leur demande à lécrit, ils ne réussissent pas. » (Enseignante 1) Elle ajoute qu'il lui est nécessaire de tenir compte, dans sa correction, du fait que certains élèves sont en 6 e année bien que certaines notions ne soient pas acquises en français. Bref, il y a « [...] beaucoup de mesures d’adaptation dans mon enseignement ». (Enseignante 1) Une autre enseignante explique qu’elle apprend de ces élèves en difficulté. En effet, «[...] j’ai appris justement à décortiquer, à renommer, à donner une procédure, il y a des enfants qui ont besoin de procédures ». (Enseignante 3)

L’enseignement par projet est aussi présent. L’enseignante 1 a intégré le français à la préparation des élèves pour le projet Expo-Sciences². Ces derniers devaient réaliser une recherche scientifique. Pour une autre enseignante, les projets transversaux amènent les élèves à des pratiques décriture dans différentes disciplines scolaires « On fait comme un transfert de compétences dans le sens qu’on est en Univers social, mais en Français on va utiliser le thème pour faire une intention d'écriture, toutes les lectures vont être reliées à cela [...]. » (Enseignante 2). Les projets transversaux constituent une occasion de pratiquer l'écriture dans le cadre du cours Éthique et culture religieuse, par exemple, et à s'habituer à justifier leurs points de vue et prises de position.

Lapprentissage par cœur, ou mémorisation, ne fait pas partie des pratiques quotidiennes, mais elles reconnaissent que certains éléments se retiennent mieux grâce à certains trucs. L’enseignante 4 explique que les trucs mnémotechniques tels que «mordre/mordu » demeurent pertinents pour retenir certaines règles. « [...] je vais continuer [...] ce qui peut les aider, mais ce n'est pas vrai que je vais tout balayer du revers de la main ce que nous on a appris ».

Les pratiques de modelage demeurent présentes dans les classes. En effet, lenseignante 1 fait beaucoup de pratiques décriture au tableau. L'enseignante 2 explique comment elle procède :

[...] jai éveillé à une notion [...] par exemple, aux légendes en leur racontant, en leur demandant den raconter [...] en écoutant Fred Pellerin ${ }^{3}$. Puis une fois que l'éveil est fait, on essaie de décortiquer cést quoi ça une légende. [...] Puis on essaie, je tombe dans le modelage dans mon

2 L'Expo-sciences est une compétition scientifique pour tous les jeunes de 6 à 20 ans. (http://exposciences.qc.ca/fr/cest-quoi)

3 Fred Pellerin est un conteur québécois (www.frepellerin.com) 
enseignement stratégique [...] de comment c’est le schéma d’un récit puis, on va sentraîner à en raconter entre nous, en lire, à en trouver sur Internet puis, ils vont se mettre à faire de la production écrite [...]. (Enseignante 2)

Pour l'enseignante 3, les pratiques de modelage se concentrent sur l'apprentissage de règles de grammaire. Quant à l'enseignante 4, le modelage va de pair avec l'utilisation du tableau interactif.

Je vais activer les connaissances antérieures. Après ça, je vais en faire une au tableau avec les enfants, en me donnant en exemple puis en réfléchissant tout haut, en me posant des questions tout haut. Après ça, je peux leur demander en équipe de deux ou de quatre, de composer des phrases ensemble pour en finir pour faire de l'individuel, pour que chacun soit capable. (Enseignante 4)

Lécriture partagée apparait comme une autre stratégie employée. Pour l'enseignante 1, cette pratique se traduit par la préparation en groupe d'une situation initiale d’un récit.

[...] on va le travailler au tableau, on va faire la correction puis on va l'enrichir puis, je vais leur dire "Qu'est-ce que tu peux écrire pour continuer l'histoire? " où je vais dire "Aujourd'hui, on fait une situation initiale. " Tout le monde a fait une situation initiale, un début. "Qu'est-ce qu'ily a dans une situation initiale?» [...]. Ça sảarrête là, mais on fait une petite pratique de situation initiale. (Enseignante 1)

\section{Discussion des résultats}

À la lumière des résultats obtenus suite à cette recherche, nous sommes en mesure d'en venir à quelques constats à l'égard des pratiques enseignantes du lire-écrire en $6^{\mathrm{e}}$ année du primaire.

Les enseignantes rencontrées utilisent des approches intégrées de l'enseignement du français. Les tâches décriture proposées font appel à leur créativité et sont généralement en lien avec les activités de lecture réalisées en classe. Elles mettent à profit létude de contes ou de légendes, par exemple, pour amener les élèves à écrire. Elles mettent également de l’avant des projets interdisciplinaires et transversaux qui amènent les élèves à faire de la recherche documentaire, à traiter l’information et l'employer dans un texte écrit. Plusieurs activités signifiantes favorisent la réflexion, l'argumentation et la rédaction de textes, mettant ainsi à profit les apprentissages réalisés en classe de français à propos des textes d’opinion. Les effets positifs de ces approches intégrées quant aux apprentissages chez les élèves sont reconnus par les enseignantes. La démarche d’appropriation des connaissances par les élèves est privilégiée par les enseignantes.

Bien que les approches intégrées fassent partie des pratiques quotidiennes des enseignants rencontrées, nous constatons cependant qu'elles conservent des pratiques appartenant aux approches dites classiques telles que la dictée. L'emploi de la dictée n’aborde cependant pas le caractère d'antan, mais selon une approche actualisée qui met les élèves en action en interrogeant leur enseignante (Dictée Zéro faute) et en mettant lévaluation au service des apprentissages. Les enseignantes organisent l'enseignement du français de manière non cloisonnée, c'est-à-dire que les activités proposées aux élèves constituent un tout. Cette méthode les aide à retenir et à s’approprier les apprentissages. Les pratiques déclarées par les enseignantes sont ainsi dans l’esprit de la réforme de l'école québécoise de 2001. Elles permettent le développement des compétences langagières et des savoirs chez les élèves les menant ainsi à la réussite dans leurs apprentissages.

\section{Conclusion et limites de la recherche}

Lanalyse des données recueillies auprès des quatre enseignantes permet d’avancer plusieurs pistes de réflexion. Les résultats amènent la nécessité d'une réflexion profonde quant à l'impact des pratiques didactiques sur l'enseignement-apprentissage du lire-écrire. Il est utile de rappeler que c'est au primaire que lélève acquiert les fondements de la langue orale et écrite. Comme les quatre enseignantes sont dans des classes considérées dans un milieu favorisé, il serait utile d’étendre létude et d'impliquer des enseignantes du milieu défavorisé. Une des pistes de recherche les plus stimulantes serait détudier l'impact des pratiques intégrées de l'enseignement du lire-écrire sur les performances des élèves aussi bien en milieu régulier quauprès des élèves présentant des difficultés d’apprentissage des compétences langagières. Ainsi, l'impact des pratiques intégrées en enseignement du lire-écrire est à mettre en lien avec la réussite des élèves et leur persévérance scolaire. Ces pistes de recherche constituent des avenues possibles permettant de nourrir la réflexion sur la place des pratiques enseignantes en classes du primaire. 
Cette recherche comporte certaines limites. En effet, les résultats produits par la recherche comportent une dimension exploratoire. En recherche qualitative, un échantillon de petite taille peut suffire et il nous a permis d'obtenir une saturation des données dans le domaine étudié. L'approche utilisée fournit des pistes à exploiter et à explorer. Une étude auprès d'un échantillon plus large d’enseignantes du primaire permettrait d'identifier plus attentivement les pratiques enseignantes privilégiées en classe d'enseignement du lire-écrire. Le choix de travailler sur les pratiques déclarées par les enseignantes nous confronte aux biais de la désirabilité sociale. Les enseignantes nous ont dit probablement des éléments de pratiques qui gagneraient à être corroborés par des observations en classe. Toutefois, létude a permis d’explorer des pratiques pédagogiques valorisées par les enseignantes participantes au projet.

\section{Références bibliographiques}

Adad, D. \& Richard-Principalli, P. (2007). Lire pour écrire, écrire pour grandir : mener des projets décriture avec des enfants de 3 à 11 ans. Lyon : Chronique sociale.

Bertrand, Y., (1993). Théories contemporaines de l'éducation, Lyon : Chronique sociale.

Bissonnette, S., Richard, M. \& Gauthier, C. (2005). Interventions pédagogiques efficaces et réussite scolaire des élèves provenant de milieux défavorisés. Revue française de pédagogie, (150), p. 87-141.

Blain, S. \& Lafontaine, L. (2010). Mettre les pairs à contribution lors du processus décriture : une analyse de l'impact du groupe de révision rédactionnelle des élèves québécois et néo-brunswickois. Revue des sciences de l'éducation, 36(2), p. 469-491.

Conseil supérieur de léducation (1999). Pour une meilleure réussite scolaire des garçons et des filles. Bulletin électronique Panorama. Document téléacessible à l'adresse :

http://www.cse.gouv.qc.ca/FR/Article/index.html?id=1999-11-001\&cat=1999-11

Debeurme, G. (2006). Difficultés d'apprentissage en écriture. Document téléacessible à l'adresse : http : // www.adaptationscolaire.org. themmes/diec/presdiec.htm

Giasson, J. (2011). La lecture. Apprentissage et difficultés.Montréal : Gaëtan Morin éditeur.

Goupil, G. (2007). Élèves en difficulté d’adaptation ou d’apprentissage (5édition). Boucherville: Gaëtan Morin éditeur.

Hall, K. (2003). Effective literacy teaching in the early years of school. Dans N. Hall, J. Larson \& J. Marsh (dir.), Handbook of Early Chilhood literacy (315-326). London : Sage.

Landry, R. (2002). Lanalyse de contenu. Dans B. Gauthier (dir.), Recherche sociale : De la problématique à la collecte de données (329-356). Sainte-Foy, Presses de l'Université du Québec, 2002, p. 329-356.

Laroui, R. (2010). Chapitre 17 : Les pratiques enseignantes face aux enjeux culturels de l'école. L'exemple du Québec ", dans R. Malet, Écoles, médiations et réformes curriculaires. Perspectives internationales (227-235). Bruxelles : De Boeck.

Laroui, R. (2008). Lapproche culturelle de l'enseignement du français et des pratiques enseignantes au secondaire dans le Bas-Saint-Laurent. Revue des sciences de léducation, 33(3), p. 371-381.

Lavoie, N., Lévesque J.-Y. \& Laroui, R. (2007). Interactions entre élèves de première année du primaire dans des situations d'écriture. Dans J.-P. Gaté \& C. Gaux (dir.), Lire-écrire, de l’enfance à lâge adulte. Rennes : Presses universitaires de Rennes.

Lefrançois, P. (2000). Apprendre à écrire à la fin du primaire : là où processus cognitifs, interdisciplinarité, coopération et hypermédia se rejoignent. Revue des sciences de léducation, 26(2), p. 325-346.

Ministère de l'Éducation, du Loisir et du Sport (2008). Mieux soutenir le développement de la compétenceà écrire. Rapport du comité d'experts sur l'apprentissage de l'écriture. Téléacessible à l’adresse: http://www.mels.gouv.qc.ca/sections/publications/index.asp?page=fiche\&id=219

Ministère de léducation du loisir et du sport (2005). Apprendre à lire. Action concertée pour le soutien à la recherche à la lecture. Téléacessible à l'adresse : http://www.mels.gouv.qc.ca/sections/publications/index.asp?page=etudes

Ministère de l'éducation du Québec (2001). Programme de formation de lécole québécoise. Éducation préscolaire et enseignement primaire. Québec: Gouvernement du Québec.

Morin, M.-F. \& Montésinos-Gelet, I. (2007). Approcher lécrit à pas de loup : la littérature de jeunesse pour apprendre à lire et écrire au préscolaire et au primaire. Montréal : Chenelière Éducation.

Mucchielli, A., (2009). Dictionnaire des méthodes qualitatives en sciences humaines et sociales. Paris : Armand Colin.

Nadon, Y. (2002). Lire et écrire en première année... et pour le reste de sa vie. Montréal : Chenelière/McGraw-Hill.

Pasa, L., Raganon, S. \& Filjalkow, J. (2006). Entrer dans lécrit avec la littérature de jeunesse. Issy-les-Moulineaux : ESF éditeur. 
Prenoveau, J. (2007). Cultiver le goût de lire et d'écrire : enseigner la lecture et lécriture par une approche équilibrée. Montréal : Chenelière Éducation.

Reuter, Y. (2011). Penser la perspective didactique : la question de l’articulation disciplinaire, pédagogique et scolaire. Dans B. Daunay, Y. Reuter \& B. Scheneuwly (dir.). Les concepts et les méthodes en didactique du français (35-54). Namur : Presses universitaires de Namur. Reuter, Y. (2000). Enseigner et apprendre à écrire. Construire une didactique de l’écriture. Paris : ESF éditeur.

Routman, R. (2009). Enseigner l'écriture : revenir à l'essentiel. Montréal : Chenelière Éducation.

Savoie-Zajc, L. (2002). L’entrevue semi-dirigée. Dans B. Gauthier (dir.), Recherche sociale. De la problématique à la collecte de données (262285). Sainte-Foy : Presses de l'Université du Québec.

Strickland, D.-S., Ganske, K. \& Monroe, J.-K. (2009). Les difficultés en lecture et en écriture. Montréal : Chenelière Éducation. Tardif, J. (1997). Pour un enseignement stratégique : L’apport de la psychologie cognitive. Montréal : Les Éditions Logiques.

Van Grunderbeeck, N., Théôret, M., Cartier, S.C. \& Chouinard, R. (2003). Étude longitudinale et transversale des conditions favorables au développement des habitudes et des compétences en lecture chez des élèves du secondaire. Rapport de recherche remis au FCAR,. Vygotski, L.V. (1985). Pensée et langage. Paris : Éditions sociales. 


\section{Annexe 1 : Canevas d'entrevue}

Phase introductive

Bonjour, je tiens d'abord à vous remercier d'avoir accepté de participer à cet entretien dans le cadre d'une recherche portant sur les pratiques denseignement de lécriture déclarées par les enseignants de sixième année du primaire menée par $\mathrm{M}^{\mathrm{me}}$ Stéphanie Leblanc en collaboration avec $\mathrm{M}^{\mathrm{me}} \mathrm{R}$ Kia Laroui, toutes deux professeures à l'université du Québec à Rimouski.

Avant de débuter l'entrevue, je vous invite à consulter et à signer le formulaire de consentement éclairé qui vous rappelle l'objectif de la recherche qui est de connaitre les pratiques d'enseignement de lécriture déclarées par les enseignants de sixième année du primaire. Le document vous informe aussi que l'entrevue, d’environ une heure, sera enregistrée et que les données seront détruites au terme de la recherche. Lanonymat est garanti. Vous êtes libre de vous retirer de la recherche en tout temps en communiquant avec la chercheuse principale aux coordonnées que vous retrouvez à la fin du document.

Avez-vous des questions ou des commentaires à formuler avant que nous commencions?

Déroulement de l'entrevue

(Les questions formulées dans le présent canevas constituent une base pour la discussion avec l'enseignant. Des questions seront sans doute ajoutées selon le fil de l'entrevue.)

\section{Sur les pratiques d'enseignement de l'écriture}

1. Comment abordez-vous l'enseignement de lécriture auprès de vos élèves de sixième année du primaire?

a. Qu'est-ce qui vous importe le plus dans cet enseignement ?

2. Quelles sont vos méthodes privilégiées d'enseignement de l'écriture?

a. Enseignement explicite (direct instruction)?

i. Modelage visant la compréhension.

ii. Pratique guidée/dirigée pour consolider les apprentissages.

iii. Pratique autonome/indépendante visant la maîtrise, l'appropriation et la rétention.

b. Projets d'écriture transversaux?

c. Utilisation de la littérature de jeunesse?

d. Écriture partagée interactive?

3. Quelle est la place réservée à l'orthographe, à la grammaire, à la syntaxe dans votre enseignement de lécriture ?

a. Qu'est-ce qui vous apparait le plus important à enseigner en écriture?

i. Orthographe?

ii. Grammaire?

iii. Syntaxe?

iv. Étapes du processus rédactionnel ?

4. Comment vous y prenez-vous pour que vos activités d'enseignement soient signifiantes pour les élèves?

a. Rapport positif à lécriture?

Sur les élèves de sixième année du primaire

5. Comment décririez-vous votre classe actuelle?

6. Votre pratique enseignante inclue-elle la pédagogie différenciée ? Si oui, de quelle manière se traduit-elle?

a. Percevez-vous des différences entre les filles et les garçons en apprentissage de l'écriture?

i. Retards?

ii. Difficultés d’apprentissage?

b. Percevez-vous une différence entre les élèves selon leur milieu familial ?

i. Famille aisée.

ii. Famille de classe moyenne.

iii. Famille défavorisée.

\section{Informations sociodémographiques}

\section{Sexe :}

Âge :

Formation :

Année d’expérience en enseignement :

Année d'expérience en $6^{\mathrm{e}}$ année du primaire :

Conclusion de lentrevue

Nous en sommes à la conclusion de l'entrevue.

Avez-vous des commentaires à ajouter suite à notre discussion?

Je vous remercie énormément du temps que vous m’avez accordé aujourd'hui. Nous en sommes très reconnaissants. Si vous souhaitez être tenu au courant des résultats de cette recherche, je vous invite à en informer la chercheuse principale. 